\title{
FUNDAMENTO, ESENCIA Y EREIGNIS. EN TORNO A LA UNIDAD DEL CAMINO DEL PENSAR DE MARTIN HEIDEGGER
}

\author{
Ángel XoLOCOTZI \\ Universidad Iberoamericana
}

\begin{abstract}
RESUMEN. Inspirado en la interpretación que hace Gadamer del camino filosófico de su maestro Heidegger, el artículo aborda el paso de la ontología fundamental (Ser y tiempo) al pensar onto-histórico (Aportes a la filosofia. Acerca del evento) a partir de la diferencia entre la esencia del Dasein y el fundamento de esa esencia. El autor presenta aquello que se mantiene así como lo que se abandona en el pensar del evento [Ereignis], y muestra como insuficiente la interpretación gadameriana que ve el giro [Kehre] de Heidegger como un regreso a sus primeras lecciones.
\end{abstract}

\section{Introducción}

El acceso al camino filosófico de un pensador presenta dificultades. Una comprensión acertada de la fenomenología-hermenéutica de Martin Heidegger no es la excepción. La Heidegger-Forschung ha interpretado de múltiples formas la obra filosófica del pensador de Meßkirch. Carl-Friedrich Gethmann ha resumido esto en dos grupos: las interpretaciones evolucionistas y las interpretaciones pluralistas. ${ }^{1}$ Las primeras, como las de Hans-Georg Gadamer o Günter Figal, ven un desarrollo «ascendente» 0 «descendente» en la obra de Heidegger ${ }^{2}$, mien-

${ }^{1}$ C.-F. GethmanN, «Philosophie als Vollzug und als Begriff. Heideggers Identitätsphilosophie des Lebens in der Vorlesung vom Wintersemester 1921/22 und ihr Verhältnis zu "Sein und Zeit"", Dilthey-Jabrbuch 4 (1986-87), pp. 27-53.

2 Cf. H.-G. GADAMER, «Erinnerungen an Heideggers Anfänge», Dilthey-Jahrbuch 4 (198687), pp. 13-26 [traducción: «Recuerdo de los comienzos de Heideggern, en Los caminos de Heidegger, Barcelona, Herder, 2002, pp. 295-306]; G. FIGAL, Heidegger zur Einfuihrung, Hamburgo, Junius Verlag, 1996, especialmente p. 49. 
tras que las segundas, como las de Otto Pöggeler o Theodore Kisiel, ven el conjunto de la obra en términos de «etapas" y "fases". ${ }^{3}$ Estos intérpretes concuerdan en dividir la obra de Heidegger en tres partes: las lecciones anteriores a Ser y tiempo [Syt], la ontología fundamental, expresada principalmente en Syt, y el pensar histórico del ser como acontecimiento o pensar del Ereignis, expresado fundamentalmente en los Beiträge zur Philosophie (Vom Ereignis). ${ }^{4}$ Gran parte de la discusión con respecto a la relación entre estos tres períodos se centra en la justificación de lo determinante de alguno de ellos y el fracaso o regresión de los otros. Gadamer tomó parte en esta discusión al considerar a Syt como una «improvisación» en donde Heidegger recae en la concepción trascendental husserliana. ${ }^{5}$ Por eso Heidegger dará el "giro" [Kehre] de la ontología fundamental de Syt al pensar del Ereignis, el cual es interpretado por Gadamer como un regreso [Rückkehr] al planteamiento de las primeras lecciones: la Kehre es un Rückkehr. ${ }^{6}$

3 Cf. O. POGGELER, "Nachwort zur zweiten Auflage», en Der Denkweg Martin Heideggers, Tubinga, Neske, 1983, p. 300 [traducción: El camino del pensar de Martin Heidegger, Madrid, Alianza, 1986, p. 367]; T. KISIEL, "Das Entstehen des Begriffsfeldes 'Faktizität' im Frühwerk Heideggers», Dilthey-Jahrbuch 4 (1986-87), pp. 91-120, especialmente pp. 113 y 116.

4 MeIdegGer, Beiträge zur Philosophie (Vom Ereignis), Fráncfort del Meno, Klostermann, 1989. Desde el 2003 contamos con una traducción al español: M. Heidegger, Aportes a la filosofia. Acerca del evento, trad. de D. V. Picotti, Buenos Aires, Biblos, 2003.

5 Además de lo que Gadamer plantea en su escrito "Erinnerungen an Heideggers Anfänge» (es importante mencionar que Gadamer habla de "Improvisation" únicamente en el texto publicado en el Dilthey-Jahrbuch, ya que en la versión publicada en sus Gesammelte Werke son suprimidas sus aseveraciones en torno a Ser y tiempo), en otros sitios indicó siempre su valoración acerca de Ser y tiempo. Por ejemplo en "La historia de la filosofía» Gadamer dice lo siguiente: "Con la disolución del marco filosofico trascendental, lo único que mantenía en pie a la herencia del idealismo, tenía que caer la historia de los problemas, que extraía sus 'problemas' de esta herencia. Esto se refleja aún en el intento de Heidegger de modificar desde el trabajo de reflexión histórica del pensamiento de Dilthey la concepción sistemática de la filosofía trascendental de su admirado maestro Husserl, el fundador de la fenomenologia, y de conseguir así una especie de síntesis entre la problemática diltheyana de la historicidad y la problemática de la ciencia de la orientación husserliana de base trascendental" (Los caminos de Heidegger, op. cit., p. 140).

${ }^{6}$ Gadamer indica esto en varios escritos: "Así, en la conversación en 1924, el 'viraje' ya estaba presente. También lo estaba, estoy convencido, ya en la primera afirmación que escuché de Heidegger en mi vida. Un joven estudiante, que había vuelto de Friburgo a Marburgo, contó con gran entusiasmo que un joven profesor había dicho desde su tarima: 'mundea' ['es weltet']. También esto fue el viraje antes del viraje" (ibíd., p. 283); "La historia enseña, como veo con una claridad cada vez mayor en las últimas décadas, que el llamado "viraje" de Heidegger en realidad sólo era el retorno a su auténtica intención, que ya había anticipado a veces en su íntima confrontación 
Pero ‘acierta la interpretación de Gadamer con respecto a la relación entre los diversos momentos de la obra de Heidegger? Considerar la Kehre solamente como Rückkebr significaría una valoración determinada de la obra de Heidegger en su totalidad que daría preeminencia indiscutible a las primeras lecciones. El pensar histórico del ser significaría únicamente el retorno al camino correcto iniciado en 1919, pero desviado en 1927 con Syt. ¿¿Una interpretación en este sentido refleja las intenciones y las modificaciones llevadas a cabo por Heidegger a lo largo de su obra? ¿No será que una interpretación de este matiz se mantiene distante del núcleo de la problemática heideggeriana?

Una constante dificultad que enfrenta todo lector en su lectura de Syt es una forma de trabajo que Heidegger llevó a cabo por lo menos en la redacción de Syt. Una investigación de los motivos de esta forma de trabajo rebasa nuestra intención actual. Baste aquí mencionarlos simplemente. Por un lado algunos temas ya tratados con antelación en otros escritos o, en este caso, lecciones, no son acentuados, ni repetidos; otros, por su parte, sólo son mencionados, ya que su análisis tendrá lugar en un momento posterior.

Con respecto a la primera presuposición del modo de redactar de Heidegger las indicaciones formales [formale Anzeige] constituyen un claro ejemplo. ${ }^{7}$ Por otra parte algunos temas anticipados o mencionados en la parte publicada de $S y t$ no son desarrollados porque su exposición estaba programada para la tercera sección: Tiempo y ser. La tematización de la diferencia ontológica y la trascendencia, sobre lo que basaremos nuestro análisis, son ejemplos de esto.

El objetivo de las reflexiones siguientes será mostrar algunos puntos que puedan contribuir a una comprensión más clara de la relación entre la ontología fundamental, en donde a mi juicio también se ubican las primeras lecciones, y el

juvenil con Husserl. Me acuerdo una y otra vez que ya en 1920 el joven Heidegger usó la expresión 'es weltet' [mundea]" (ibíd., p. 303).

${ }^{7}$ A partir de la lectura de Ser y tiempo parecería que la indicación formal es dejada completamente de lado en este texto. Pero en una carta a Karl Löwith en 1927 Heidegger escribe lo siguiente: «Indicación formal, crítica de la doctrina común del a priori, formalización, etcétera, todo está para mí ahí, aun cuando ahora no hablo de ello" (M. HEIDEGGER, "Drei Briefe Martin Heideggers an Karl Löwith", en D. Papenfuss y O. Pöggeler [ed.], Zur philosophischen Aktualität Heideggers, vol. 2, Fráncfort del Meno, Klostermann, 1990, p. 37). 
pensar histórico del ser. ${ }^{8}$ Con esto pretendo destacar en cierto sentido la unidad del pensar de Martin Heidegger.'

\section{Diferencia ontológica y horizonte trascendental}

Como se sabe la expresión más acabada de la ontología fundamental se lleva a cabo en Syt. Sin embargo, conviene aclarar que Syt puede entenderse en tres sentidos fundamentales: Syt en sentido estrecho se refiere a la primera y segunda secciones, publicadas en 1927: Etapa preparatoria del análisis fundamental del Dasein y Dasein y temporalidad. Syt en sentido amplio se refiere por su parte a toda la obra, tal y como estaba programada en el $\$ 8$ de la primera sección. ${ }^{10}$ Con Syt en sentido más amplio nos referimos a la co-pertenencia trascendental-horizontal entre ser y Dasein. ${ }^{11}$

Así pues la tematización de la diferencia ontológica y del horizonte trascendental expresamente se ubican en Syt en sentido amplio, pero no en sentido estrecho. Por ello encontramos su despliegue en la lección de verano de 1927 Los problemas fundamentales de la fenomenologia ${ }^{12}$ y posteriormente en el artículo de 1929 dedicado a Husserl: De la esencia del fundamento. ${ }^{13}$

Que el lugar expositivo de la diferencia ontológica lo ubiquemos en los textos mencionados no significa que en la parte publicada de Syt se encuentre completamente ausente: ahí simplemente es mencionada, pero su desarrollo tendría

${ }^{8}$ Cf. mi texto Der Umgang als «Zugang». Der hermeneutisch-phänomenologischer "Zugang» zum faktischen Leben in den frühen Freiburger Vorlesungen Martin Heideggers, Berlín, Duncker und Humblot, 2002. Una parte reformulada de este trabajo apareció en español: Fenomenología de la vida fáctica. Heidegger y su camino a Ser y tiempo, México, Plaza y Valdés-UIA, 2004.

9 Con respecto a la unidad del pensar de Heidegger, cf. O. Cosmus, Anonyme Phänomenologie: Die Einheit von Heideggers Denkweg, Wurzburgo, Konnigshausen und Neumann, 2001.

${ }^{10}$ M. HeIDEgGer, Sein und Zeit (a partir de ahora, citado como SuZ), Tubinga, Niemeyer, 1993, pp. 39-40 [Ser y tiempo (a partir de ahora, citado como Syt), Madrid, Trotta, 2003, pp. 62-63].

${ }^{11}$ Cf. F.-W. von HerRmann, "Wahrheit-Zeit-Raum», en E. Richter (ed.), Die Frage nach der Wabrheit, Fráncfort del Meno, Klostermann, 1997, p. 144.

${ }^{12}$ M. HeIDEGGer, Gesamtausgabe (a partir de ahora, citada como GA), vol. 24, Fráncfort del Meno, Klostermann, 1989 [Los problemas fundamentales de la fenomenologia, Madrid, Trotta, 2000].

${ }^{13}$ M. HeIDEGGER, Wegmarken, GA vol. 9, Fráncfort del Meno, Klostermann, 1976. [Hitos, Madrid, Alianza, 2000]. 
lugar en la tercera sección. Ya es conocido que la primera versión de la tercera sección Tiempo y ser fue destruida por el mismo Heidegger. La mencionada lección de 1927 constituye según Heidegger mismo la nueva versión de la parte destruida:

Pero la sección propiamente «sistemática" acerca de Tiempo y ser fue insuficiente en la primera versión y a la vez circunstancias externas (el aumento del volumen del anuario) impidieron, por suerte, la publicación de esta parte, con respecto a la cual no había ninguna firme confianza al saber de la insuficiencia. El intento fue deshecho, pero a la vez, más por caminos históricos, se hizo una nueva vía en la lección del semestre de verano de $1927 .^{14}$

Ahora bien, en esa lección Heidegger expone una comprensión horizontal de la diferencia ontológica. Esto significa que para poder comportarme con lo entornado (lo experimentado a partir del entorno o mundo circundante) en mi trato procurador-comprendedor necesito llevar a cabo una diferencia. La vivencia del entorno [Umwelterlebnis] debe mantenerse esencialmente en este diferenciar: en la diferencia entre la apertura de ser que ocurre en la comprensión de ser y el descubrimiento de los entes que ocurre en el comportarse con ellos. Mientras que en las primeras lecciones y en Syt (en sentido estrecho) el acento se halla en el develamiento del Dasein comprendedor de ser en tanto que ser-en-el-mundo, es decir, el acento recae principalmente en la comprensión de mundo; el ser de lo que no es existencia ${ }^{15}$, y con respecto a lo cual el Dasein se comporta, es desarrollado expresamente en la tercera sección Tiempo y ser. Con la tematización del ser de lo que no es existencia se prepara a la vez la base para la concreta tematización de la diferencia ontológica.

En este punto debemos evitar considerar la diferencia ontológica como un nuevo tema de la filosofía de Heidegger. Más bien la diferencia ontológica en el carácter de poder diferenciar está ya presupuesta en los análisis de la vivencia del mundo circundante, aun cuando no es destacada expresamente.

${ }^{14}$ M. HeidegGer, Besinnung, GA vol. 66, pp. 413-14 (el subrayado es nuestro). Cf. F.-W. VON HerRmanN, Heideggers "Grundprobleme der Phänomenologie", Zur "Zweiten Hälfte" von "Sein und Zeit" ", Fráncfort del Meno, Klostermann, 1991 [Traducción: La segunda mitad de Ser y tiempo. Sobre Los Problemas Fundamentales de la Fenomenologia de Heidegger, Madrid, Trotta, 1997].

${ }_{15}$ «Existencia», entendido como el modo de ser específico del ser-humano; cf. el $\$ 9$ de Syt. 
El preguntar explicitante de Heidegger con respecto a esta diferencia ontológica conduce a la pregunta por el poder del diferenciar. Ya que el poder-diferenciar debe ser pensado como poder ser existente, Heidegger escribe en De la esencia del fundamento que el fundamento del poder diferenciar debe ser buscado en el fundamento del poder ser existente ${ }^{16}$. En este sentido la vida fáctica $o$ Dasein comprendedor de ser debe tener «la raíz de su propia posibilidad» en el fundamento de su esencia.

Pero la raíz de la posibilitación no debe ser buscada en la esencia de la vida fáctica o Dasein, sino en el fundamento de la esencia del Dasein. Por ello podemos diferenciar entre la esencia del Dasein y el fundamento de la esencia del Dasein. Lo primero es llamado, en el $\$ 9$ de Syt, existencia, mientras que lo segundo es caracterizado en De la esencia del fundamento como trascendencia. ${ }^{17}$ Pasemos ahora a considerar este nexo en forma más detallada.

En el $\$ 9$ de Syt Heidegger escribe: «La 'esencia' del Dasein se halla en su existencia»" ${ }^{18}$. En el párrafo anterior en lugar de existencia escribe tener-que-ser [zusein]. Que el Dasein tiene su esencia existenciaria ${ }^{19}$ en el tener-que-ser debe ser visto en su doble estructura: en el proyecto yecto o en la entregada relación de ser. En el $\S 4$ de Syt ya se ha anticipado aquello que posteriormente será caracterizado como proyecto [Entwurf]: «a este ente le va en su ser este mismo ser. La constitución de ser del Dasein implica entonces que el Dasein tiene en su ser una relación de ser con su ser ${ }^{20}$. Que el Dasein tiene que ser [zu-sein] debe entenderse por un lado como esta relación con su ser, es decir, como proyecto. Pero que yo en mi ser tenga una relación de ser con mi ser no está determinado a par-

${ }^{16}$ GA 9, p. 135 [Hitos, p. 118].

${ }^{17}$ Cf. ibíd., y también GA 24 [Los problemas fundamentales de la fenomenologia], pp. 88 y ss. Heidegger despliega un análisis muy iluminador de la trascendencia en su lección invernal de 1928/29, Einleitung in die Philosophie, GA 27 [traducción: Introducción a la filosofia, Madrid, Cátedra, 1999], particularmente en los parágrafos 28, 29, 36-38.

${ }_{18}$ SuZ, p. 42 [Syt, p. 67].

${ }^{19}$ Con esencia existenciaria quiero referirme al concepto específico de esencia al que Heidegger se refiere por medio de las comillas en la palabra "esencia". Esencia existenciaria mienta un concepto de esencia, formado únicamente en función de la existencia como un modo de ser del hombre, no en virtud del concepto de esencia tradicional como essentia, el cual denota la coseidad categorial de las cosas. Por ello al hablar de esencia existenciaria en Heidegger debe diferenciarse siempre del concepto de esencia categorial-esencial de la tradición.

${ }^{20}$ SuZ, p. 12 [Syt, p. 34]. 
tir de mí, sino que me encuentro puesto, colocado en esta relación de ser: estoy entregado, dado a mi ser en tanto que relación de ser. Dicho de otra forma: en tanto que soy, soy de tal manera en este yo-soy que debo comportarme en mi ser con mi ser en forma ejecutante. Y este tener que comportarme ejecutoriamente remite al otro sentido de tener-que-ser $[z u$-sein $]$ : al estar yecto.

En mi tener-que-comportarme-en-mi-ser-con-mi-ser, es decir, en mi existencia, me comporto en mi ser de tal forma que mediante este comportamiento el ser, que comprendo en mi comprensión de ser, está abierto. Pero esta apertura de ser no es solamente la apertura de mi modo de ser, de mi existencia, sino a la vez del ser de los entes que no son existencia, con los cuales me comporto en mi comportamiento descubridor. Al escribir una ponencia no sólo comprendo mi ser en tanto que relación entregada de ser, sino a la vez el ser de los entes que no son existencia como la pluma o el escritorio, como seres-a-la-mano, como útiles. Por ello Von Herrmann habla adecuadamente de una doble apertura de ser: por un lado de la apertura de mi existencia como una apertura extática-misma y por otro lado de la apertura de ser de los entes que no son existencia como una apertura horizontal. ${ }^{21}$ Esta apertura integral se esconde en el término 'Dasein': en el 'Da-' de 'Dasein' debe pensarse la apertura integral del ser en general, mientras que en '-sein' se mienta la existencia en tanto que apertura extática. En una anotación marginal a la palabra 'Sein' en el $\$ 9$ de Syt Heidegger se refiere a ello: "¿Cuál? [El que consiste en] Tener que ser el Ahí y en él afirmarse ante el Ser en cuanto tal»"

Ya hemos indicado que en nuestro comportamiento cotidiano llevamos a cabo la diferencia entre esta doble apertura y el descubrimiento de los entes, a saber, la diferencia ontológica como poder diferenciar. También hemos anticipado que el preguntar de Heidegger se dirige al fundamento posibilitador de este poder diferenciar. De hecho este será encontrado en el fundamento de la esencia del Dasein: en la trascendencia en el sentido de sobrepasar.

El Dasein en su poder diferenciar, en su existir comprendedor de ser, ya es sobrepasador: sobrepasa al ente en dirección a la apertura de ser y retorna desde p. 141.

${ }^{21}$ F.-W. vON Herrmann, Hermeneutik und Reflexion, Fráncfort del Meno, Klostermann, 2000, 22 SuZ, p. 440, anotación marginal a de la página 42 [Syt, p. 67, anotación marginal $c$ ]. 
esta apertura yecta-proyectada de ser a los entes en el descubrir pre-predicativo del ente. Sólo hasta que este movimiento trascendental se ha llevado a cabo en su totalidad es que podemos encontrar al ente como ente, descubrirlo patentemente.

Formalmente podemos aprehender el sobrepasar del trascender en una relación tripolar: de, hacia y algo, es decir, de algo hacia algo y en donde algo es sobrepasado. El de mienta aquello que sobrepasa, lo trascendental, a saber, el Dasein mismo. El hacia es el «sobre el que» hacia lo que sobrepasa el Dasein: la apertura de ser. Aquello, algo, que es sobrepasado es el ente pero aún no como ente; sólo en el regreso que pertenece a la trascendencia es que el ente es descubierto como ente.

A partir de lo expuesto podemos ver que para poder descubrir al ente como ente en mi trato procurador, requiero tanto de la apertura del ser de este ente, así como del movimiento sobrepasador del existir. Lo primero lo hemos caracterizado como la apertura horizontal $\mathrm{u}$ horizonte, lo segundo como el movimiento trascendental o trascendencia. Trascendencia y horizonte son pues los conceptos con los cuales Heidegger piensa en un primer intento, ontológico-fundamental, el fundamento de la esencia del Dasein. Dicho de otra forma: el horizonte trascendental es el fundamento ontológico-fundamental de la esencia del Dasein.

En el horizonte trascendental Heidegger piensa, como destaca Von Herrmann, la copertenencia ontológica-fundamental de Dasein y ser: en el horizonte se muestra la relación de la verdad del ser con la existencia del Dasein, mientras que en la trascendencia se muestra el comportamiento esencial de la existencia con la verdad del ser. ${ }^{23}$

En este punto debemos destacar algunos elementos centrales desarrollados hasta aquí: 1) Debemos diferenciar entre la pregunta por la esencia de la vida fáctica o Dasein y la pregunta por el fundamento de la esencia del Dasein. 2) La pregunta por la esencia de la vida fáctica o Dasein tiene como punto de partida las primeras lecciones y encuentra su concreción en la analítica existenciaria del Dasein en Syt (en sentido estrecho). 3) La pregunta por el fundamento de la esencia del Dasein pertenece expresamente a la temática de la tercera Sección de Syt. Tiempo y ser. 4) El horizonte trascendental en tanto que fundamento de la esen-

${ }^{23}$ F.-W. vON HERrManN, "Daseinsanalyse und Ereignisdenken", Daseinsanalyse 12 (1995), pp. 6-17, aquí p. 8. 
cia del Dasein es pues la interpretación ontológica-fundamental de la copertenencia de Dasein y ser.

\section{El paso de la ontología fundamental al pensar del Ereignis}

Si mantenemos a la vista la diferenciación entre la esencia del Dasein y el fundamento de la esencia del Dasein, podemos comprender en forma más clara el segundo planteamiento de la pregunta por el ser que Heidegger lleva a cabo. En otras palabras: podemos utilizar esta diferenciación como medio auxiliar para observar de modo más cercano el paso de la ontología fundamental al pensar histórico del ser como Ereignis.

Anteriormente hemos hecho referencia a interpretaciones que se basan en "fases" o "cambios de tema" con respecto al camino filosófico de Heidegger. Otros comentaristas, por su parte ven el pensar histórico del ser como un nuevo intento de Heidegger que anula lo anterior por completo. Este punto de vista no ve ninguna continuidad en su trabajo filosófico, sino únicamente dos propuestas filosóficas aisladas, en donde la segunda, el pensar histórico del ser, exige el abandono de la primera, la ontología fundamental.

Empero, una interpretación más detallada, tanto vertical como horizontal, del camino filosófico de Heidegger exige no un simple abandono unilateral, sino una investigación explícita de ambas perspectivas de la pregunta por el ser. Tal investigación muestra que Heidegger no abandona ni rechaza la ontología fundamental en general, sino solamente la interpretación horizontal-trascendental de la comprensión de ser. ${ }^{24}$ Con otras palabras: los análisis obtenidos en la elaboración ontológica-fundamental de la pregunta por la esencia del Dasein no son abandonados, sino solamente la elaboración en torno a la pregunta por el fundamento de la esencia del Dasein, es decir, el horizonte trascendental.

${ }^{24}$ Agradecemos esta concepción fundamental a F.-W. von Herrmann, quien ha mostrado en diferentes trabajos lo fundamental en este respecto. El paso de la ontología fundamental al pensar histórico del ser ya lo había tratado en 1961 en su tesis doctoral Die Selbstinterpretation Martin Heideggers. Allí, él realizó una investigación pionera, a pesar de la restringida literatura (Los problemas fundamentales de la fenomenología, y las Beiträge aún no se habian publicado); investigación en la cual expuso adecuadamente la continuidad del camino del pensar de Heidegger. La literatura secundaria de su época no alcanzó a ver esto. Se limitaba a hablar de «quiebre* o "rompimiento". Cf. también F.-W. vON HERRMANN, Wege ins Ereignis, Fráncfort del Meno, Klostermann, 1994, p. 6. 
Heidegger mismo indicó que lo que abandonó en el pensar histórico del ser fue el horizonte trascendental. En las Beiträge zur Philosophie (Vom Ereignis) escribe lo siguiente: "Puesto que, pues, el Da-sein como Da-sein sostiene originariamente lo abierto de la ocultación, rigurosamente tomado no se puede hablar de una trascendencia del Da-sein; en el circuito de este planteo la representación de 'trascendencia' tiene que desaparecer en todo sentido" ${ }^{25}$. En una anotación marginal a Syt Heidegger escribe en relación con la planeada sección Tiempo y ser lo siguiente: "La superación del horizonte en cuanto tal. Reversión al origen. El estar-presente desde este origen ${ }^{26}$.

Las estructuras obtenidas del Dasein que se desplegaron en torno a la pregunta por su esencia no desaparecen al abandonar el fundamento ontológicofundamental, el horizonte trascendental. Más bien son reinterpretadas a partir de un preguntar más originario por el fundamento de la esencia en la perspectiva histórica del ser. Si en la ontología fundamental la esencia del Dasein tiene su fundamento en la trascendencia y en el horizonte, ahora en el pensar histórico del ser se devela su fundamento acontecedor. En el pensar histórico del ser la verdad del ser en tanto que lance acontecedor, ahora experimentada históricamente, se encuentra explicitada en relación con el Dasein comprendedor de ser, el cual se entiende como Dasein arrojado y acontecido. La relación de la verdad para el Dasein ahora es pensada como lance. El Dasein está yecto desde este lance de la verdad del ser en la verdad lanzante del ser.

El tránsito de la ontología fundamental al pensar histórico del ser fue caracterizado por Heidegger como vuelta, giro o viraje [Kehre]. Ya Von Herrmann y Coriando han mostrado los diversos sentidos en que Heidegger emplea el término Kehre, ${ }^{27}$ de modo que la usual expresión «Heidegger antes y después de la vuelta» es ambigua en gran medida. ${ }^{28}$ En este punto sólo nos referimos a la «vuel-

${ }^{25}$ GA 65, p. 217. Traducción de Dina V. Picotti, aunque conservo el término Da-sein, traducido por ella como ser-ahí.

${ }^{26} S u Z$ p. 440, anotación marginal $b$ de la página 39 [Syt, p. 62, anotación marginal b].

27 F.-W. von HerRMANN, Wege ins Ereignis, op. cit., pp. 64-84; P.-L. CORIANDO, Der letzte Gott als Anfang, Múnich, Fink, 1998, \$4-6. Además cf. T. KALARIPARAMBIL, Das befindliche Verstehen und die Seinsfrage, Berlín, Duncker und Humblot, 1999, $\$ 30$; I. AUGSBERG, Wiederbringung des Seienden. Studien zur ontologischen Differenz im seinsgeschichtlichen Denken Martin Heideggers, Múnich, Fink, 2003, $\$ 7 \mathrm{~b}$ y $9 \mathrm{c}$.

${ }^{28}$ Cf. F.-W. von HerRmann, Heideggers Philosophie der Kunst, Fráncfort del Meno, Klostermann, 1994, p. XIV (Prólogo). 
ta” en el sentido de tránsito de la ontología fundamental al pensar histórico del ser y no a sus otros significados. ${ }^{29}$

Así pues, la vuelta o giro en tanto que tránsito de la ontología fundamental al pensar histórico del ser debe ser entendido sólo como el abandono del fundamento ontológico-fundamental, es decir, el horizonte trascendental y como el tomar el fundamento acontecedor. ${ }^{30}$ Una tematización más exhaustiva del giro [Kehre] la dejaremos para otra ocasión. Por el momento basta indicar que una adecuada comprensión de la Kehre impide dos problemas interpretativos bastante extendidos en los interpretes de Heidegger: 1) Como ya insinuamos, el giro no puede ser entendido como sinónimo o prueba del "fracaso" de la ontología fundamental. 2) Si uno aprueba esta interpretación, entonces puede caer en otra, igualmente problemática, que propone considerar el giro como un simple regreso a los planteamientos descubiertos en las primeras lecciones. ${ }^{31}$ Una interpreta-

29 1: El paso de Syt (1. y 2." Secciones) a la 3. Sección, Tiempo y ser, 2: La vuelta en la metontología u óntica metafísica.

${ }^{30}$ Esto mismo lo confirma Heidegger en "Protocolo de un seminario sobre Tiempo y sem, (1962): “Dado pues que el fundamento de la ontología fundamental no es ningún fundamento sobre el que pudiera construirse, no es fundamentum inconcussum alguno, sino mucho más un fundamentum concussum; y dado asimismo que la reiteración de la analítica del estar humano [Dasein] es ya parte integrante del planteamiento básico de Ser y tiempo, mientras que la palabra 'fundamento' contradice el carácter precursor de dicha analítica, de ahí que se haya abandonado el título 'ontología fundamental'" (M. HeIDEgGER, Tiempo y ser, Madrid, Tecnos, 1999, p. 51).

31 T. Kisiel es quien representa ambos malentendidos. Como ejemplo tomemos una cita de un texto que aparece en 1996: "With the Turn, the exaggerated claims for the scientific Charakter of Philosophy give way to the judgment that the book Being and Time was an aberrant way to the one topic of philosophy and thought. When Heidegger first realizes that Being and Time was a failed project, he ten re-turns to earlier insights left unpursued in order to begin again. This Return is the real meaning of his self-professed and much discussed 'Turn'. Thus the theme of es weltet first sounded in 1919 resounds again 1929: das Ereignis reappears in the following decade with an ever increasing insistance. The Book of Genesis of Being and Time must therefore conclude by following the same trajectory, going beyond Being and Time by going back to its most incipient beginning in Kriegsnotsemester 1919" ("The Genesis of Being and Time, The Primal Leap", en L. Langsdorf y S. Watson [ed.], Phenomenology, Interpretation, and Community, Albany, State University of New York, p. 53).

SuZ no puede ser considerado por ningún motivo como un "proyecto fallido". Que aparezca un cambio en la perspectiva no significa que la primera perspectiva, en la cual se enmarca $S u Z$, deba ser abolida o considerada como un "camino aberrante". En el pensar histórico del ser la pregunta por el ser es entendida de un modo mucho más claro, aunque se abandone la perspectiva horizontal-trascendental de la ontología fundamental. Esto no significa que las estructuras funda- 
ción tal pasa de largo las diferencias fundamentales y los acentos temáticos que Heidegger desarrolló a lo largo de sus textos y lecciones.

Para concluir diremos lo siguiente: con lo expuesto hemos querido mostrar que lo que Heidegger tematiza en las primeras lecciones y en Syt remite a la esencia de la vida fáctica o Dasein y no expresamente al fundamento de la esencia del Dasein. Como ya indicamos, la tematización expresa de este fundamento pertenece a la temática de la tercera Sección de Syt. Este fundamento es abandonado en el pensar histórico del ser. Sin embargo, la esencia del Dasein, es decir, las estructuras esenciales de la existencia, no son abandonadas, sino reinterpretadas originariamente al ser experimentado el estado de yecto del Dasein en su origen desde el lance acontecedor. En este sentido la Kehre no puede ser vista como un simple regreso a los planteamientos iniciales, como piensa Gadamer, ya que lo expuesto desde 1919 con respecto a la esencia de la vida fáctica o Dasein no fue abandonado nunca. ¿Cómo se puede regresar a algo nunca abandonado? De este modo se puede ver que lo determinante de la fenomenología-hermenéutica de Martin Heidegger se mantuvo en toda su obra y constituye una unidad en el camino de su pensar.

mentales del Dasein, las cuales son descubiertas en la ontología fundamental, simplemente deben ser vistas como un "fracaso". Más bien ellas se mantienen en la nueva perspectiva, están, sin embargo, interpretadas de una forma más originaria. Desafortunadamente muchos intérpretes no ven este hecho fundamental y siguen moviéndose en el camino fácil y superficial del "fracaso". Un cuestionamiento de esta forma de ver el camino filosófico de Heidegger lo hacen las siguientes interpretaciones: F.-W. vON HERRMANN, Die Selbstinterpretation Martin Heideggers; Wege ins Ereignis, pp. 5-64, "Wahrheit-Zeit-Raum», «Daseynanalyse und Ereignisdenken»; P. EMAD, "Heidegger I, Heidegger II, and Beiträge zur Philosophie (Vom Ereignis)», en B. E. Babich (ed.), From Phenomenology to Thought, Errancy and Desire: Essays in Honor of William J. Richardson, S.J., Dordrecht, Kluwer, 1995, pp. 129-146; «A Conversation with Friedrich-Wilhelm von. Herrmann on Heidegger's 'Beiträge zur Philosophie' ', en B. Hopkins (ed.), Phenomenology: Japanese and American Perspectives, Dordrecht, Kluwer, 1999, pp. 145-166. 